\title{
Analysis of the cystic fibrosis transmembrane conductance regulator (CFTR) gene, in patients with alcoholic liver disease (ALD) and alcoholic chronic pancreatitis (ACP)
}

\author{
Gangwani $\mathbf{A}^{1}$, Jain $\mathbf{M K}^{2}$ \\ ${ }^{1}$ Dr Amar Gangwani, Assistant Professor, Department of Pathology, Bundelkhand Medical College, Sagar, MP, ${ }^{2}$ Dr \\ Manish Kumar Jain, Department of Zoology, Dr .H.S. Gaur University, Sagar, MP, India
}

Address of Correspondence: Dr Amar Gangwani, Email: drgangwaniamar79@gmail.com

\begin{abstract}
Introduction: Cystic fibrosis $(\mathrm{CF})$ is an autosomal recessive disorder affecting multiple organs. A defective CFTR gene leads to inadequate transport of $\mathrm{Cl}$ ions between intra- and extracellular environment of cells in affected organs. Susceptibility to alcoholic chronic pancreatitis (ACP) could be genetically determined. Mutations in cystic fibrosis transmembrane conductance regulator $(C F T R)$ genes have been variably associated with both hereditary and idiopathic form of chronic pancreatitis (CP). Our aim was to analyze these genes in ACP patients. Mutational screening was performed in 05 unrelated ACP patients and 05 patients with alcoholic liver disease (ALD). Method: Patients with ACP and ALD, were admitted in Bundelkhand Medical College hospital, Sagar, and enrolled for genetic analysis. Genomic DNA was extracted from whole blood according to the established protocols using the DNA Isolation Kit for Mammalian blood (Genei Bangalore). Results: Mutation analysis of CFTR was performed in all ACP and ALD patients. In three ACP patients, $\triangle \mathrm{F} 508$ mutation was detected in heterozygous state with a prevalence rate of $8.88 \%$. R117 $\mathrm{H}$ was another mutation detected in ACP patients in heterozygous state. Conclusion: Present study was performed to determine whether patients with ACP and ALD had mutations in the CFTR gene and to explore whether non coding sequences that produce low levels of CFTR mRNA (the 5T allele) was responsible for above mentioned abnormalities. Our hypothesis was that the pancreatic damage due to high alcohol intake could be due to abnormal allele or a combination of multiple mutations occurring in the two alleles in CFTR gene.
\end{abstract}

Keywords: Alcohol, Cystic Fibrosis, Alcoholic Chronic Pancreatitis (ACP), Acute Liver Disease (ALD)

\section{Introduction}

Cystic Fibrosis (CF) is an autosomal recessive genetic disease that was first described in 1936 by the Swiss pathologist, Guido Fanconi, who reported the autopsy and clinical characteristics of three patients with bronchiectasis and pancreatic insufficiency [1]. In 1938, Dr Dorothy Anderson published an autopsy study of 38 infants, described the findings as "cystic fibrosis of the pancreas" and recognized the syndrome as an inherited disease [2].

Chronic pancreatitis (CP) is a potentially lifethreatening disease characterized by a progressive inflammatory disorder ultimately leading to irreversible

Manuscript received: $6^{\text {th }}$ Nov 2015

Reviewed: $14^{\text {th }}$ Nov 2015

Author Corrected: $30^{\text {th }}$ Nov 2015

Accepted for Publication: $11^{\text {th }}$ Dec 2015 morphological changes and permanent impairment of exocrine and endocrine functions. Most patients with CP suffer from relapsing attacks of abdominal pain and are at a markedly increased risk of developing maldigestion, diabetes mellitus, and pancreatic cancer [3]. Alcoholism is the most common etiologic factor in up to $70 \%$ of patients with CP. Other causes include drugs, duct-obstructing lesions, and metabolic or autoimmune disorders [2]. In the rare form of hereditary pancreatitis (HP), at least two major mutations $(R 122 H$ and $N 29 I$ ) in the cationic trypsinogen gene (protease, serine 1, PRSS1) have been identified. In vitro biochemical studies suggest that these two mutations and additional pancreatitis-associated PRSS1 mutations [4] may inhibit autolysis of trypsin and or enhance autoactivation of trypsinogen, resulting in a gain of trypsin. 
In up to $30 \%$ of patients, association with any of the aforementioned factors is lacking and the disease is classified as idiopathic (ICP). Some evidence exists that at least in a small proportion of patients with ICP, a mutation of one or both alleles of either the cystic fibrosis transmembrane conductance regulator (CFTR) gene $[5,6]$. Severe mutations in both alleles of the CFTR results in the commonly recognized cystic fibrosis (CF) having clinical features of abnormal sweat chloride concentrations, pancreatic insufficiency, and progressive pulmonary disease. Among CF patients, two-thirds have a deletion of three-base pair between the nucleotides 1652 and 1655 with subsequent deletion of the phenylalanine amino acid at codon $508(\Delta \mathbf{F 5 0 8}$, Fig.1), although approximately 1000 other mutations have been reported. Most CFTR mutations can be classified according to a 15 severity category system based on the presumed or demonstrated molecular consequences. Typical CF patients with severe pancreatic impairment tend to have two severe mutations (i.e, class I, II, or III), whereas CF patients with pancreatic sufficiency from birth tend to have at least one CF 'mild allele' (ie, class IV or V).(7)].

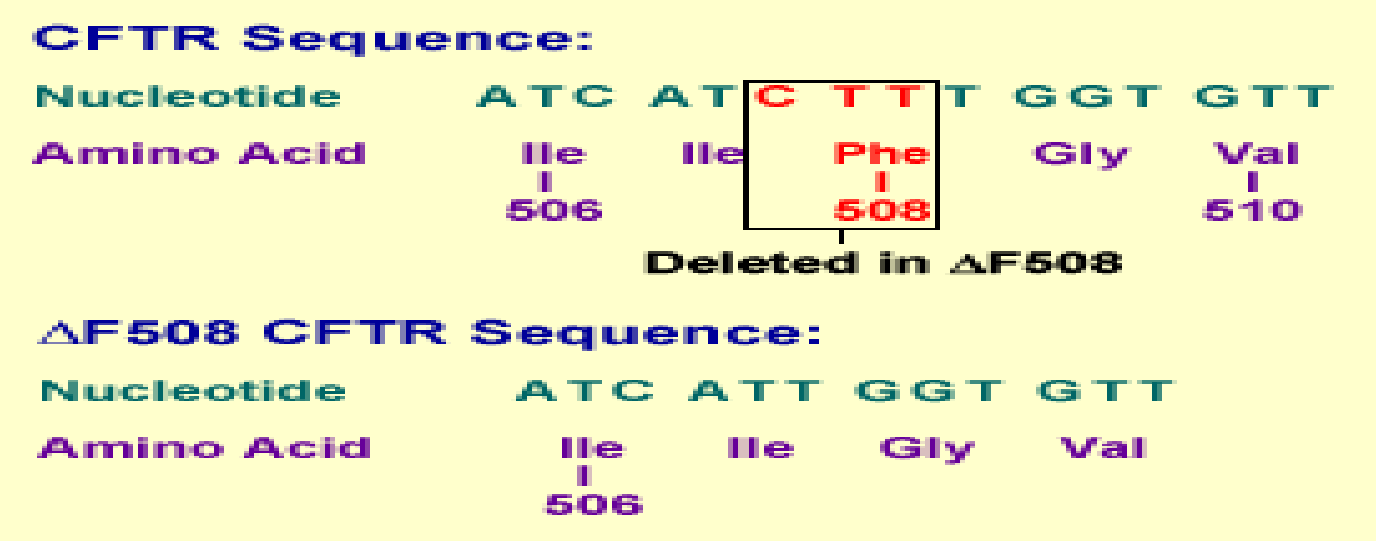

Figure 1: The $\Delta$ F508 deletion is the most common cause of cystic fibrosis. The isoleucine (Ile) at amino acid position 507 remains unchanged because both ATC and ATT code for isoleucine

SPINK1 is a peptide that is synthesized by pancreatic acinar cells and co localizes with trypsinogen in the zymogen granules. SPINK1 acts as the first line of defence against prematurely activated trypsinogen in the acinar cells by physically blocking the active site of trypsin. The most known mutation $(N 34 S)$ of the SPINK1 gene is relatively common (up to $4 \%$ of general population) and markedly increased (up to 25\%) in ICP patients [7]. The discovery of gene mutations that induce or predispose to $\mathrm{CP}$ led some researchers to investigate about a possible causal role of genetic factors in the occurrence and development of alcoholic chronic pancreatitis (ACP). Evidence for a genetic basis for ACP comes from epidemiological, laboratory, and clinical studies: only about 5-10\% alcoholics suffer from clinically recognized CP. Although a linear correlation exists between the risk of developing CP and the quantity of alcohol consumption, there is no apparent threshold of toxicity. Long-term, high-dose alcohol feeding of laboratory animals fails to cause CP. To date, most of the relevant studies in ACP performed mutation analysis of one single gene that is CFTR. Moreover, due to differences in the selection and number of participants and the mutation screening method used, the reported mutation rates observed in one single gene i.e CFTR, varied greatly among different studies. A simultaneous analysis of all three genes in patients with ACP would provide insights into the relative contribution of each gene to the etiology of this disease.

The aim of this study was to perform this kind of analysis by screening the most relevant mutations of the CFTR genes in patients (of Sagar District) with ACP. As controls, we screened patients from the same geographical area who were affected by alcoholic liver disease (ALD) without a clinically recognized pancreatic disease.

\section{Materials and Methods}

Patients with ACP and ALD, who were consecutively admitted in Govt. District Hospital associated hospital of Bundelkhand Medical College, Sagar were enrolled for genetic analysis. 
DNA Extraction: Genomic DNA was extracted from whole blood according to the established protocols using the DNA Isolation Kit for Mammalian blood (Genei Bangalore).

Mutation Screening of the CFTR gene: Most frequent mutation ( $\Delta F 508)$ was examined with the polymerase chain reaction (PCR) followed by an ARMS. Identification of unknown mutations was done by Single Strand Conformational Polymorphism (SSCP) analysis (Applied Biosytems). The CFTR polymorphic intron 8 poly $\mathrm{T}$ region was analyzed according to the method described by Chillon et al [7].

Statistical Analysis: Student's $t$-test was used for age, age at symptoms' onset, symptoms' duration, and alcohol consumption. Pearson's $x^{2}$-test was used for sex and smoking habits. Comparison of mutation frequency between ACP and ALD patients was performed by means of Fisher's exact test (expected values lower than 5 per cell). A $P$-value less than 0.05 were considered to indicate statistical significance.

\section{Results}

Patients: A total of 10 unrelated patients (05 males; mean age: 38 years) with ACP and 05 patients ( males; mean age: 44 years) with ALD were studied. We did not find any significant difference between the two groups of patients as far as alcohol consumption and smoking habits was considered. Furthermore, no specific association was observed between any demographic or clinical subjects' characteristics and gene mutations (Table 1).

Table 1: Sequence variations identified in the CFTR genes in 05 ACP patients

\begin{tabular}{|c|c|c|}
\hline \multicolumn{3}{|c|}{ CFTR } \\
\hline Patient & Mutation & IVS8 (Poly T variant) \\
\hline 1 & $\mathrm{R} 117 \mathrm{H}$ & $\mathrm{U}$ \\
\hline 2 & $\Delta \mathrm{F} 508$ & 7T/9T \\
\hline 3 & $\Delta \mathrm{F} 508$ & $7 \mathrm{~T} / 7 \mathrm{~T}$ \\
\hline 4 & $\mathrm{R} 117 \mathrm{H}$ & $7 \mathrm{~T} / 7 \mathrm{~T}$ \\
\hline 5 & $\Delta \mathrm{F} 508$ & $7 \mathrm{~T} / 7 \mathrm{~T}$ \\
\hline
\end{tabular}

U- Unidentified

Table 2: Sequence variations identified in the CFTR genes in 05 ALD patients.

\begin{tabular}{|l|l|l|}
\hline \multicolumn{1}{|c|}{ CFTR } & Mutant & Poly T \\
\hline Patient & - & ND \\
\hline 1 & - & $7 / 7$ \\
\hline 2 & N1303K & $7 / 7$ \\
\hline 3 & & $5 / 7$ \\
\hline 4 & - & $5 / 5$ \\
\hline 5 & & \\
\hline
\end{tabular}

$\mathrm{ND}$, not done due to insufficient DNA sample

Mutation analysis of the CFTR gene: Mutation analysis of CFTR was performed in all ACP patients and in all ALD patients. In three ACP patients, $\triangle \mathrm{F} 508$ mutation was detected in heterozygous state (Table 1) with a prevalence rate of $8.88 \%$. This prevalence was different from the expected prevalence of $3.22 \%(P=0.64)$ in our geographical area. $\mathrm{R} 117 \mathrm{H}$ was another mutation detected in two ACP patients in heterozygous conditions. No patient with ACP carrying CFTR mutations had the $5 T$ allele (Table1). A single mutation, N1303K, in the heterozygous state, was detected in one ALD patient (suffering from $\mathrm{CP}$ ) with a prevalence rate $(3.03 \%)$ more than that expected in the general population. 


\section{Discussion}

Moreover, one would speculate that an alcoholic drinker who simultaneously carries a major mutation of the CFTR gene in one allele and a minor mutation of the same gene in the other allele is likely to be more affected by an atypical form of CF with CP than ACP. In the present study, the prevalence of mutations of CFTR in ACP patients was similar to that observed in ALD and more than expected in normal population from the same geographical area

The lack of a significant difference in the prevalence of gene mutations between ACP and ALD patients could be affected by a beta error due to the small sample size.

The main objectives of this study was to determine whether patients with Acute Chronic Pancreatitis (ACP) and Acute Liver Disease (ALD) had mutations in the CFTR gene and to explore whether non coding sequences that produce low levels of CFTR mRNA (the $5 T$ allele) was responsible for above mentioned abnormalities. In the present study, our hypothesis was that the pancreatic damage due to high alcohol intake could be due to abnormal allele or a combination of multiple mutations occurring in the two alleles in CFTR gene (compound heterozygote). This hypothesis has already been successfully tested in both ICP and tropical calcific pancreatitis (TCP). The latter is an idiopathic, juvenile, nonalcoholic form of $\mathrm{CP}$ widely prevalent in several tropical countries. By simultaneously analyzing 39 ICP subjects for common mutations of CFTR, SPINK1, and PRSS1, Noone et al (2001) (8) found that about $60 \%$ of their patients had at least one mutation in either CFTR or SPINK1, or both. Interestingly, the risk of pancreatitis was increased approximately five-fold by having one CFTR mutation, 20-fold by having SPINK1 N34S mutation, 40-fold by having two CFTR mutations (compound heterozygotes), and 900-fold by having N34S and two CFTR mutations. By using a similar approach, [9] found that at least $30 \%$ of 39 French patients with ICP carried at least one abnormal allele in one of the three genes, with a compound heterozygote state for CFTR in four patients and a trans-heterozygote state for SPINK1/CFTR genes in other three patients. The $5 T$ allele causes reduced levels of normal CFTR mRNA [10], this DNA variant would appear likely to be involved in the pathogenesis of ACP and ALD. Future studies should be designed with large sample sizes including healthy controls in order to avoid potential shortcomings.

\section{Conclusion}

The pathogenesis of ACP is still an unresolved problem. Several different theories have been made such as duct obstruction by protein plugs, direct toxicity of ethanol, and oxidative stress. However, none of these mechanisms has yet found a solid experimental support to gain wide acceptance. As already mentioned, evidence for a genetic basis for ACP comes from epidemiological, laboratory and clinical studies. Although susceptibility to alcoholic pancreatic damage could be inherited, until now no clear association between any gene mutation(s) and occurrence of ACP in alcoholics has been found. In particular, during the last few years, three single genes (PRSS1, CFTR and SPINK1) have been investigated in patients with ACP.

In summary, this study shows that mutations in CFTR genes are occasionally found in patients with ACP, but their prevalence is not significantly increased in comparison with alcoholics who develop ALD without clinical, biochemical, or radiological signs of CP. Regarding the $5 \mathrm{~T}$ allele, our data are in agreement with the findings of Sharer et al. (1998) and Cohn et al. (1998) and indicate that this allele does not confer a significant risk of chronic pancreatitis. The agreement of the these studies is important from a public health standpoint because the $5 \mathrm{~T}$ allele is the most common disease associated polymorphism in the CFTR gene described to data in the general population. The role of CFTR in chronic pancreatitis is as yet unknown. Studies performed so far have not explored the whole gene and have focused on mutations involved in Cystic Fibrosis.

\section{Funding:Nil. Conflict of interest: Nil. Permission for IRB: Yes}

\section{References}

1. Fanconi, G., Wehlinger, E. and Knauer C. Das (1936) Cocliakie-Syndrom bei bronchiectasien. Wien Med Wochenschr 86, 753-756.

2. Anderson, D.H. (1938). Cystic fibrosis of the pancreas and its relation to celiac disease: A clinical and pathological study. Am. J. Dis. Child 56, 344-349 
3. Williams SG, Westaby D, Tanner MS, Mowat AP. Liver and biliary problems in cystic fibrosis. Br Med Bull. 1992 Oct;48(4):877-92.

4. C.F.F. (2003, 2004,2006) Cystic Fibrosis Foundation Patient registry. Annual Data Report. Toronto, Canada.

5. Sharer N, Schwarz M, Malone G, Howarth A, Painter J, Super M, Braganza J. Mutations of the cystic fibrosis gene in patients with chronic pancreatitis. N Engl J Med. 1998 Sep 3;339(10):645-52.

6. Cohn JA, Strong TV, Picciotto MR, Nairn AC, Collins FS, Fitz JG. Localization of the cystic fibrosis transmembrane conductance regulator in human bile duct epithelial cells. Gastroenterology. 1993 Dec;105(6):1857-64.

7. Chillón M, Casals T, Mercier B, Bassas L, Lissens W, Silber S, Romey MC, Ruiz-Romero J, Verlingue

$\mathrm{C}$, Claustres $\mathrm{M}$, et al. Mutations in the cystic fibrosis gene in patients with congenital absence of the vas deferens. N Engl J Med. 1995 Jun 1;332(22):1475-80.

$\begin{array}{lrrr}\text { 8.Noone } & \text { PG, Zhou } & \text { Z, Silverman } & \text { LM, Jowell } \\ \text { PS, Knowles } & \text { MR, Cohn } & \text { JA. } & \text { Cystic }\end{array}$ fibrosis gene mutations and pancreatitis risk: relation to epithelial ion transport and trypsin inhibitor genemutations. Gastroenterology. 2001 Dec;121(6):1310-9.

9. Audrézet MP, Chen JM, Le Maréchal C et al: Determination of the relative contribution of three genes, the cystic fibrosis transmembrane conductance regulator gene, the cationic trypsinogen gene, and the pancreatic secretory trypsin inhibitor gene, to the etiology of idiopathic chronic pancreatitis. Eur J Hum Genet 2002; 10: 100-106.

10. Chu CS, Trapnell BC, Curristin S, Cutting GR, Crystal RG. Genetic basis of variable exon 9 skipping in cystic fibrosis transmembrane conductance regulator mRNA. Nat Genet. 1993 Feb;3(2):151-6.

\section{How to cite this article?}

Gangwani A, Jain MK. Analysis of the cystic fibrosis transmembrane conductance regulator (CFTR) gene, in patients with alcoholic liver disease (ALD) and alcoholic chronic pancreatitis (ACP). Int J Med Res Rev 2015;3(11):1353-1357. doi: 10.17511/ijmrr.2015.i11.245. 